\title{
The Existence of Solutions for Four-Point Coupled Boundary Value Problems of Fractional Differential Equations at Resonance
}

\author{
Yumei Zou, ${ }^{1}$ Lishan Liu, ${ }^{2}$ and Yujun $\mathrm{Cui}^{3}$ \\ ${ }^{1}$ Department of Statistics and Finance, Shandong University of Science and Technology, Qingdao 266590, China \\ ${ }^{2}$ School of Mathematical Sciences, Qufu Normal University, Qufu, Shandong 273165, China \\ ${ }^{3}$ Department of Mathematics, Shandong University of Science and Technology, Qingdao, Shandong 266590, China
}

Correspondence should be addressed to Yujun Cui; cyj720201@163.com

Received 18 December 2013; Accepted 14 February 2014; Published 23 March 2014

Academic Editor: Xinguang Zhang

Copyright (C) 2014 Yumei Zou et al. This is an open access article distributed under the Creative Commons Attribution License, which permits unrestricted use, distribution, and reproduction in any medium, provided the original work is properly cited.

A four-point coupled boundary value problem of fractional differential equations is studied. Based on Mawhin's coincidence degree theory, some existence theorems are obtained in the case of resonance.

\section{Introduction}

In this paper, we are concerned with the following four-point coupled boundary value problem for nonlinear fractional differential equation. Consider

$$
\begin{gathered}
D_{0+}^{\alpha} u(t)=f\left(t, u(t), D_{0+}^{\alpha-1} u(t), v(t), D_{0+}^{\beta-1} v(t)\right), \\
D_{0+}^{\beta} v(t)=g\left(t, u(t), D_{0+}^{\alpha-1} u(t), v(t), D_{0+}^{\beta-1} v(t)\right), \\
\left.I_{0+}^{2-\alpha} u(t)\right|_{t=0}=0, \quad u(1)=a v(\xi), \\
\left.I_{0+}^{2-\beta} v(t)\right|_{t=0}=0, \quad v(1)=b u(\eta),
\end{gathered}
$$

where $1<\alpha, \beta<2, D_{0+}^{\alpha}$ and $I_{0+}^{\alpha}$ are the standard RiemannLiouville differentiation and integration, $f, g \in C([0,1] \times$ $\left.\mathbb{R}^{4}, \mathbb{R}\right), a, b \in \mathbb{R}, \xi, \eta \in(0,1)$, and

$$
a b \xi^{\beta-1} \eta^{\alpha-1}=1
$$

The subject of fractional calculus has gained considerable popularity and importance because of its intensive development of the theory of fractional calculus itself and its varied applications in many fields of science and engineering. As a result, the subject of fractional differential equations has attracted much attention; see [1-11] for a good overview.

At the same time, we notice that coupled boundary value problems, which arise in the study of reaction-diffusion equations and Sturm-Liouville problems, have wide applications in various fields of sciences and engineering, for example, the heat equation [12-14] and mathematical biology [15, 16]. In [17], Asif and Khan used the Guo-Krasnosel'skii fixedpoint theorem to show the existence of positive solutions to the nonlinear differential system with coupled four-point boundary value conditions

$$
\begin{aligned}
-x^{\prime \prime}(t) & =f(t, x(t), y(t)), \quad t \in(0,1), \\
-y^{\prime \prime}(t) & =g(t, x(t), y(t)), \quad t \in(0,1), \\
x(0) & =y(0)=0, \\
x(1) & =\alpha y(\xi), \quad y(1)=\beta x(\eta),
\end{aligned}
$$

where $\xi, \eta \in(0,1), 0<\alpha \beta \xi \eta<1$, and $f, g:(0,1) \times[0,+\infty) \times$ $[0,+\infty) \rightarrow[0,+\infty)$ are two given continuous functions.

In [18], the authors considered the existence of positive solutions of four-point coupled boundary value problem for 
systems of the nonlinear semipositone fractional differential equation

$$
\begin{gathered}
D_{0+}^{\alpha} u+\lambda f(t, u, v)=0, \quad t \in(0,1), \lambda>0, \\
D_{0+}^{\alpha} v+\lambda g(t, u, v)=0, \\
u^{(i)}(0)=v^{(i)}(0)=0, \quad 0 \leq i \leq n-2, \\
u(1)=a v(\xi), \quad v(1)=b u(\eta),
\end{gathered}
$$

where $\lambda$ is a parameter, $a, b, \xi, \eta$ satisfy $\xi, \eta \in(0,1), 0<$ $a b \xi \eta<1, \alpha \in(n-1, n]$ is a real number and $n \geq 3$, and $D_{0+}^{\alpha} u$ is Riemann-Liouville's fractional derivative.

Recently, Cui and Sun [19] showed the existence of positive solutions of singular superlinear coupled integral boundary value problems for differential systems

$$
\begin{gathered}
-x^{\prime \prime}(t)=f_{1}(t, x(t), y(t)), \quad t \in(0,1), \\
-y^{\prime \prime}(t)=f_{2}(t, x(t), y(t)), \quad t \in(0,1), \\
x(0)=y(0)=0, \quad x(1)=\alpha[y], \quad y(1)=\beta[x],
\end{gathered}
$$

where $\alpha[x], \beta[x]$ are bounded linear functionals on $C[0,1]$ given by

$$
\alpha[x]=\int_{0}^{1} x(t) d A(t), \quad \beta[x]=\int_{0}^{1} x(t) d B(t)
$$

with $A, B$ being functions of bounded variation with positive measures.

A key assumption in the above papers is that the case studied is not at resonance; that is, the associated fractional (or ordinary) linear differential operators are invertible. In this paper, instead, we are interested in the resonance case due to the critical condition (2). Boundary value problems at resonance have been studied by several authors including the most recent works [20-31]. In this paper, we establish new results on the existence of a solution for the couple boundary value problems at resonance. Our method is based on the coincidence degree theorem of Mawhin [32, 33].

Now, we briefly recall some notations and an abstract existence result.

Let $Y, Z$ be real Banach spaces and let $L: \operatorname{dom} L \subset Y \rightarrow$ $Z$ be a Fredholm operator of index zero. $P: Y \rightarrow Y$ and $Q: Z \rightarrow Z$ are continuous projectors such that

$$
\begin{aligned}
& \operatorname{Im} P=\operatorname{Ker} L, \quad \operatorname{Ker} Q=\operatorname{Im} L, \\
& Y=\operatorname{Ker} L \oplus \operatorname{Ker} P, \quad Z=\operatorname{Im} L \oplus \operatorname{Im} Q .
\end{aligned}
$$

It follows that $\left.L\right|_{\operatorname{dom} L \cap \operatorname{Ker} P}: \operatorname{dom} L \cap \operatorname{Ker} P \rightarrow \operatorname{Im} L$ is invertible. We denote the inverse of the mapping by $K_{P}$ (generalized inverse operator of $L$ ). If $\Omega$ is an open bounded subset of $Y$ such that $\operatorname{dom} L \cap \Omega \neq \emptyset$, the mapping $N: Y \rightarrow Z$ will be called $L$-compact on $\bar{\Omega}$ if $Q N(\bar{\Omega})$ is bounded and $K_{P}(I-Q) N: \bar{\Omega} \rightarrow Y$ is compact.

Theorem 1 (see $[32,33])$. Let $L$ be a Fredholm operator of index zero and let $N$ be L-compact on $\bar{\Omega}$. Assume that the following conditions are satisfied. (i) $L x \neq \lambda N x$ for every $(x, \lambda) \in[(\operatorname{dom} L \backslash \operatorname{Ker} L) \cap \partial \Omega] \times$ $(0,1)$.

(ii) $N x \notin \operatorname{Im} L$ for every $x \in \operatorname{Ker} L \cap \partial \Omega$.

(iii) $\operatorname{deg}\left(\left.Q N\right|_{\text {Ker } L}, \operatorname{Ker} L \cap \Omega, 0\right) \neq 0$, where $Q: Z \rightarrow Z$ is a projector as above with $\operatorname{Im} L=\operatorname{Ker} Q$.

Then the equation $L x=N x$ has at least one solution in $\operatorname{dom} L \cap$ $\bar{\Omega}$.

For convenience, let us set the following notations:

$$
\begin{aligned}
\Delta_{1}=\max \left\{1+\frac{1}{\Gamma(\beta)},\left(1+\frac{1}{\Gamma(\alpha)}\right)\right. & \\
& \left.\times\left(1+a \xi^{\beta-1} \Gamma(\alpha)\left[\frac{b}{\Gamma(\alpha)}+\frac{1}{\Gamma(\beta)}\right]\right)\right\}, \\
\Delta_{2}= & \frac{\Gamma(\beta+1) \Gamma(\alpha+1)}{\alpha \xi^{\beta-1} \Gamma(\alpha+1)(\xi-1)+\Gamma(\beta+1)(\eta-1)}, \\
\Delta_{3}= & \frac{1}{\Gamma(\beta)} \max \left\{a \xi^{\beta-1}(\Gamma(\alpha)+1), \Gamma(\beta)+1\right\}, \\
\Delta_{4}= & \max \left\{\Delta_{1}\left\|a_{1}\right\|_{1}+\Delta_{3}\left\|a_{2}\right\|_{1}, \Delta_{1}\left\|b_{1}\right\|_{1}+\Delta_{3}\left\|a_{2}\right\|_{1}\right\}, \\
\Delta_{5}= & \max \left\{\Delta_{1}\left\|c_{1}\right\|_{1}+\Delta_{3}\left\|c_{2}\right\|_{1}, \Delta_{1}\left\|d_{1}\right\|_{1}+\Delta_{3}\left\|d_{2}\right\|_{1}\right\} .
\end{aligned}
$$

\section{Preliminaries and Lemmas}

In this section, first we provide recall of some basic definitions and lemmas of the fractional calculus, which will be used in this paper. For more details, we refer to books $[1,2,4]$.

Definition 2 (see $[1,4]$ ). The Riemann-Liouville fractional integral of order $\alpha>0$ of a function $u:(0, \infty) \rightarrow \mathbb{R}$ is given by

$$
I_{0+}^{\alpha} u(t)=\frac{1}{\Gamma(\alpha)} \int_{0}^{t}(t-s)^{\alpha-1} u(s) d s,
$$

provided that the right-hand side is pointwise defined on $(0, \infty)$.

Definition 3 (see $[1,4]$ ). The Riemann-Liouville fractional derivative of order $\alpha>0$ of a continuous function $u$ : $(0, \infty) \rightarrow \mathbb{R}$ is given by

$$
D_{0^{+}}^{\alpha} u(t)=\frac{1}{\Gamma(n-\alpha)}\left(\frac{d}{d t}\right)^{n} \int_{0}^{t} \frac{u(s)}{(t-s)^{\alpha-n+1}} d s,
$$

where $n-1 \leq \alpha<n$, provided that the right-hand side is pointwise defined on $(0, \infty)$.

We use the classical Banach space $C[0,1]$ with the norm $\|u\|_{\infty}=\max _{t \in[0,1]}|u(t)|$ and $L^{1}[0,1]$ with the norm $\|u\|_{1}=$ $\int_{0}^{1}|u(t)| d t$. We also use the space $A C^{n}[0,1]$ defined by

$$
A C^{n}[0,1]=\left\{u:[0,1] \longrightarrow \mathbb{R} \mid u^{(n-1)}\right.
$$

are absolutely continuous on $[0,1]\}$ 
and the Banach space $C^{\mu}[0,1](\mu>0)$

$$
\begin{aligned}
C^{\mu}[0,1] & \\
= & \left\{u(t) \mid u(t)=I_{0+}^{\mu} x(t)+c_{1} t^{\mu-1}+c_{2} t^{\mu-2}+\cdots\right. \\
& +c_{N-1} t^{\mu-(N-1)}, x \in C[0,1], t \in[0,1] \\
& \left.c_{i} \in \mathbb{R}, i=1,2, \ldots, N=[\mu]+1\right\}
\end{aligned}
$$

with the norm $\|u\|_{C^{\mu}}=\left\|D_{0+}^{\mu} u\right\|_{\infty}+\cdots+\left\|D_{0+}^{\mu-(N-1)} u\right\|_{\infty}+\|u\|_{\infty}$.

Lemma 4 (see [1]). Let $\alpha>0, n=[\alpha]+1$. Assume that $u \in$ $L^{1}(0,1)$ with a fractional integration of order $n-\alpha$ that belongs to $A C^{n}[0,1]$. Then the equality

$$
\left(I_{0+}^{\alpha} D_{0+}^{\alpha} u\right)(t)=u(t)-\sum_{i=1}^{n} \frac{\left.\left(\left(I_{0+}^{n-\alpha} u\right)(t)\right)^{(n-i)}\right|_{t=0}}{\Gamma(\alpha-i+1)} t^{\alpha-i}
$$

holds almost everywhere on $[0,1]$.

In the following lemma, we use the unified notation of both for fractional integrals and fractional derivatives assuming that $I_{0+}^{\alpha}=D_{0+}^{-\alpha}$ for $\alpha<0$.

Lemma 5 (see [1]). Assume that $\alpha>0$; then

(i) let $k \in \mathbb{N}$. If $D_{a+}^{\alpha} u(t)$ and $\left(D_{a+}^{\alpha+k} u\right)(t)$ exist, then

$$
\left(D^{k} D_{a+}^{\alpha}\right) u(t)=\left(D_{a+}^{\alpha+k} u\right)(t)
$$

(ii) if $\beta>0, \alpha+\beta>1$, then

$$
\left(I_{a+}^{\alpha} I_{a+}^{\beta}\right) u(t)=\left(I_{a+}^{\alpha+\beta} u\right)(t)
$$

satisfies at any point on $[a, b]$ for $u \in L_{p}(a, b)$ and $1 \leq$ $p \leq+\infty$;

(iii) let $u \in C[a, b]$. Then $\left(D_{a+}^{\alpha} I_{a+}^{\alpha}\right) u(t)=u(t)$ holds on $[a, b]$

(iv) note that, for $\lambda>-1, \lambda \neq \alpha-1, \alpha-2, \ldots, \alpha-n$, one has

$$
D^{\alpha} t^{\lambda}=\frac{\Gamma(\lambda+1)}{\Gamma(\lambda-\alpha+1)} t^{\lambda-\alpha}, \quad D^{\alpha} t^{\alpha-i}=0, \quad i=1,2, \ldots, n \text {. }
$$

Remark 6. If $1<\alpha<2$ and $u$ satisfies $D_{0+}^{\alpha} u=f(t) \in L^{1}(0,1)$ and $\left.I_{0+}^{2-\alpha} u\right|_{t=0}=0$, then $u \in C^{\alpha-1}[0,1]$. In fact, with Lemma 4 , one has

$$
u(t)=I_{0+}^{\alpha} f(t)+c_{1} t^{\alpha-1}+c_{2} t^{\alpha-2}
$$

Combined with $\left.I_{0+}^{2-\alpha} u\right|_{t=0}=0$, there is $c_{2}=0$. So

$$
u(t)=I_{0+}^{\alpha} f(t)+c_{1} t^{\alpha-1}=I_{0+}^{\alpha-1}\left[I_{0+}^{1} f(t)+c_{1} \Gamma(\alpha)\right] .
$$

Lemma 7 (see [34]). $F \subset C^{\mu}[0,1]$ is a sequentially compact set if and only if $F$ is uniformly bounded and equicontinuous. Here to be uniformly bounded means that there exists $M>0$ such that for every $u \in F$

$$
\|u\|_{C^{\mu}}=\left\|D_{0+}^{\mu} u\right\|_{\infty}+\cdots+\left\|D_{0+}^{\mu-(N-1)} u\right\|_{\infty}+\|u\|_{\infty}<M
$$

and to be equicontinuous means that for all $\epsilon>0, \exists \delta>0$ and for all $t_{1}, t_{2} \in[0,1],\left|t_{1}-t_{2}\right|<\delta, u \in F$, and $i \in\{1, \ldots,[\mu]\}$, the following holds:

$$
\left|u\left(t_{1}\right)-u\left(t_{2}\right)\right|<\varepsilon, \quad\left|D_{0+}^{\mu-i} u\left(t_{1}\right)-D_{0+}^{\mu-i} u\left(t_{2}\right)\right|<\varepsilon .
$$

We also use the following two Banach spaces $Y=$ $C^{\alpha-1}[0,1] \times C^{\beta-1}[0,1]$ with the norm

$$
\|(x, y)\|_{Y}=\max \left\{\|x\|_{C^{\alpha-1}},\|y\|_{C^{\beta-1}}\right\}
$$

and $Z=L^{1}[0,1] \times L^{1}[0,1]$ with the norm

$$
\|(x, y)\|_{Z}=\max \left\{\|x\|_{1},\|y\|_{1}\right\} .
$$

Let the linear operator $L: \operatorname{dom} L \subset Y \rightarrow Z$ with

$$
\begin{gathered}
\operatorname{dom} L=\left\{(u, v) \in Y:\left.I_{0+}^{2-\alpha} u(t)\right|_{t=0}=0, u(1)=a v(\xi),\right. \\
\left.\left.I_{0+}^{2-\beta} v(t)\right|_{t=0}=0, v(1)=b u(\eta)\right\}
\end{gathered}
$$

be defined by

$$
L(u, v)=\left(L_{1} u, L_{2} v\right),
$$

where $L_{1}: C^{\alpha-1}[0,1] \rightarrow L^{1}[0,1]$ and $L_{2}: C^{\beta-1}[0,1] \rightarrow$ $L^{1}[0,1]$ are defined by

$$
L_{1} u=D_{0+}^{\alpha} u(t), \quad L_{2} v=D_{0+}^{\beta} v(t) .
$$

Let the nonlinear operator $N: Y \rightarrow Z$ be defined by

$$
(N(u, v))(t)=\left(N_{1}(u, v)(t), N_{2}(u, v)(t)\right),
$$

where $N_{1}, N_{2}: Y \rightarrow L^{1}[0,1]$ are defined by

$$
\begin{aligned}
& N_{1}(u, v)(t)=f\left(t, u(t), D_{0+}^{\alpha-1} u(t), v(t), D_{0+}^{\beta-1} v(t)\right), \\
& N_{2}(x, y)(t)=g\left(t, u(t), D_{0+}^{\alpha-1} u(t), v(t), D_{0+}^{\beta-1} v(t)\right) .
\end{aligned}
$$

Then four-point coupled boundary value problems (1) can be written as

$$
L(u, v)=N(u, v) .
$$

Lemma 8. Let $L$ be the linear operator defined as above. If (2) holds, then

$$
\begin{gathered}
\operatorname{Ker} L=\left\{(u, v) \in \operatorname{dom} L:(u, v)=c\left(a \xi^{\beta-1} t^{\alpha-1}, t^{\beta-1}\right),\right. \\
c \in \mathbb{R}, t \in[0,1]\},
\end{gathered}
$$

$$
\begin{aligned}
\operatorname{Im} L=\{ & (x, y) \in Z: a I_{0+}^{\beta} y(\xi)-I_{0+}^{\alpha} x(1) \\
& \left.+a \xi^{\beta-1}\left[b I_{0+}^{\alpha} x(\eta)-I_{0+}^{\beta} y(1)\right]=0\right\} .
\end{aligned}
$$


Proof. Let $u(t)=a \xi^{\beta-1} t^{\alpha-1}$ and let $v(t)=t^{\beta-1}$. Then by Lemma 5, we have $\left.I_{0+}^{2-\alpha} u(t)\right|_{t=0}=\left.I_{0+}^{2-\beta} v(t)\right|_{t=0}=0, u(1)=$ $a \xi^{\beta-1}=a v(\xi), v(1)=1=b u(\eta)$, and $D_{0+}^{\alpha} u(t)=D_{0+}^{\beta} v(t)=0$. So

$$
\left\{(u, v) \in \operatorname{dom} L:(u, v)=c\left(a \xi^{\beta-1} t^{\alpha-1}, t^{\beta-1}\right), c \in \mathbb{R}\right\}
$$

C Ker $L$.

For every $(u, v) \in \operatorname{Ker} L$, if $D_{0+}^{\alpha} u(t)=D_{0+}^{\beta} v(t)=0$, then

$$
u(t)=c_{1} t^{\alpha-1}+c_{2} t^{\alpha-2}, \quad v(t)=c_{3} t^{\beta-1}+c_{4} t^{\beta-2}
$$

Considering that $\left.I_{0+}^{2-\alpha} u(t)\right|_{t=0}=\left.I_{0+}^{2-\beta} v(t)\right|_{t=0}=0, u(1)=a v(\xi)$ and $v(1)=b u(\eta)$, we can obtain that $c_{2}=c_{4}=0$ and $c_{1}: c_{3}=$ $a \xi^{\beta-1}$. It yields the following:

$$
\begin{aligned}
\operatorname{Ker} L \subset\{(u, v) \in \operatorname{dom} L:(u, v) \\
\left.=c\left(a \xi^{\beta-1} t^{\alpha-1}, t^{\beta-1}\right), c \in \mathbb{R}\right\} .
\end{aligned}
$$

Let $(x, y) \in \operatorname{Im} L$; then there is $(u, v) \in \operatorname{dom} L$ such that $(x, y)=L(u, v)$; that is, $u \in C^{\alpha-1}[0,1], D_{0+}^{\alpha} u(t)=x(t)$ and $v \in C^{\beta-1}[0,1], D_{0+}^{\beta} v(t)=y(t)$. By Lemma 4 ,

$$
\begin{aligned}
I_{0+}^{\alpha} x(t)= & u(t)-\frac{\left.\left(\left(D_{0+}^{\alpha-1} u\right)(t)\right)\right|_{t=0}}{\Gamma(\alpha)} t^{\alpha-1} \\
& -\frac{\left.\left(\left(I_{0+}^{2-\alpha} u\right)(t)\right)\right|_{t=0}}{\Gamma(\alpha-1)} t^{\alpha-2}, \\
I_{0+}^{\beta} y(t)= & v(t)-\frac{\left.\left(\left(D_{0+}^{\beta-1} v\right)(t)\right)\right|_{t=0}}{\Gamma(\beta)} t^{\beta-1} \\
& -\frac{\left.\left(\left(I_{0+}^{2-\beta} v\right)(t)\right)\right|_{t=0}}{\Gamma(\beta-1)} t^{\beta-2}
\end{aligned}
$$

and by the couple boundary conditions, we have

$$
\begin{aligned}
& u(t)=I_{0+}^{\alpha} x(t)+c_{1} t^{\alpha-1}, \quad v(t)=I_{0+}^{\beta} y(t)+c_{2} t^{\beta-1}, \\
& u(1)=I_{0+}^{\alpha} x(1)+c_{1}=a v(\xi)=a\left[I_{0+}^{\beta} y(\xi)+c_{2} \xi^{\beta-1}\right], \\
& v(1)=I_{0+}^{\beta} y(1)+c_{2}=b u(\eta)=b\left[I_{0+}^{\alpha} y(\eta)+c_{1} \eta^{\alpha-1}\right] .
\end{aligned}
$$

It yields the following:

$$
a I_{0+}^{\beta} y(\xi)-I_{0+}^{\alpha} x(1)+a \xi^{\beta-1}\left[b I_{0+}^{\alpha} x(\eta)-I_{0+}^{\beta} y(1)\right]=0 .
$$

On the other hand, suppose that $(x, y) \in Z$ satisfy (36). Let $u(t)=I_{0+}^{\alpha} x(t)+a \xi^{\beta-1} t^{\alpha-1}$ and $v(t)=I_{0+}^{\beta} y(t)+\left[b I_{0+}^{\alpha} x(\eta)-\right.$ $\left.I_{0+}^{\beta} y(1)+1\right] t^{\beta-1}$, and then $D_{0+}^{\alpha} u(t)=x(t), D_{0+}^{\beta} v(t)=y(t)$, and

$$
\begin{aligned}
& \left.I_{0+}^{2-\alpha} u(t)\right|_{t=0}=\left.I_{0+}^{2-\beta} v(t)\right|_{t=0}=0, \\
u(1)= & I_{0+}^{\alpha} x(1)+a \xi^{\beta-1} \\
= & a I_{0+}^{\beta} y(\xi)+a\left[b I_{0+}^{\alpha} x(\eta)-I_{0+}^{\beta} y(1)+1\right] \xi^{\beta-1} \\
= & a v(\xi), \\
v(1)= & I_{0+}^{\beta} y(1)+\left[b I_{0+}^{\alpha} x(\eta)-I_{0+}^{\beta} y(1)+1\right] \\
= & b I_{0+}^{\alpha} x(\eta)+a b \xi^{\beta-1} \eta^{\alpha-1}=b u(\eta) .
\end{aligned}
$$

Therefore, (30) holds.

Lemma 9. If (2) holds, then $L$ is a Fredholm operator of index zero and $\operatorname{dim} \operatorname{Ker} L=\operatorname{codim} \operatorname{Im} L=1$. Furthermore, the linear operator $K_{p}: \operatorname{Im} L \rightarrow \operatorname{dom} L \cap \operatorname{Ker} P$ can be defined by

$$
\begin{aligned}
K_{P} & (u, v)(t) \\
& =\left\{I_{0+}^{\alpha} u(t)-a \xi^{\beta-1} t^{\alpha-1}\left[b I_{0+}^{\alpha} u(\eta)-I_{0+}^{\beta} v(1)\right], I_{0+}^{\beta} v(t)\right\} .
\end{aligned}
$$

Also

$$
\left\|K_{p}(u, v)\right\|_{Y} \leq \Delta_{1}\|(u, v)\|_{Z}
$$

Proof. Define operator $Q: Z \rightarrow Z$ as follows:

$$
Q(u, v)=\Delta_{2} Q_{1}(u, v)(1,1),
$$

where $Q_{1}: Z \rightarrow \mathbb{R}$ is defined by

$$
\begin{aligned}
Q_{1}(u, v)= & a I_{0+}^{\beta} v(\xi)-I_{0+}^{\alpha} u(1) \\
& +a \xi^{\beta-1}\left[b I_{0+}^{\alpha} u(\eta)-I_{0+}^{\beta} v(1)\right], \\
\Delta_{2}= & \frac{\Gamma(\beta+1) \Gamma(\alpha+1)}{\alpha \xi^{\beta-1} \Gamma(\alpha+1)(\xi-1)+\Gamma(\beta+1)(\eta-1)} \neq 0 .
\end{aligned}
$$

It is easy to see that $Q^{2}(u, v)=Q(u, v)$; that is, $Q: Z \rightarrow Z$ is a continuous linear projector. Furthermore, $\operatorname{Ker} Q=\operatorname{Im} L$. For $(u, v) \in Z$, set $(u, v)=[(u, v)-Q(u, v)]+Q(u, v)$. Then $(u, v)-Q(u, v) \in \operatorname{Ker} Q$ and $Q(u, v) \in \operatorname{Im} Q$. It follows from $\operatorname{Ker} Q=\operatorname{Im} L$ and $Q^{2}(u, v)=Q(u, v)$ that $\operatorname{Im} L \cap \operatorname{Im} Q=(0,0)$. So we have

$$
Z=\operatorname{Im} L \oplus \operatorname{Im} Q .
$$

Now, $\operatorname{Ind} L=\operatorname{dim} \operatorname{Ker} L-\operatorname{codim} \operatorname{Im} L=\operatorname{dim} \operatorname{Ker} L-$ $\operatorname{dim} \operatorname{Im} Q=0$, and so $L$ is a Fredholm operator of index 0 . 
Let $P: Y \rightarrow Y$ be continuous linear operator defined by

$$
P(u, v)=\frac{D_{0+}^{\beta-1} v(0)}{\Gamma(\beta)}\left(a \xi^{\beta-1} t^{\alpha-1}, t^{\beta-1}\right) .
$$

Obviously, $P$ is a linear projector and

$$
\operatorname{Ker} P=\left\{(u, v) \in Y: D_{0+}^{\beta-1} v(0)=0\right\} .
$$

It is easy to know that $Y=\operatorname{Ker} P \oplus \operatorname{Ker} L$.

Define $K_{P}: \operatorname{Im} L \rightarrow \operatorname{dom} L \cap \operatorname{Ker} P$ by

$$
\begin{aligned}
& K_{P}(u, v)(t) \\
& \quad=\left(I_{0+}^{\alpha} u(t)-a \xi^{\beta-1} t^{\alpha-1}\left[b I_{0+}^{\alpha} u(\eta)-I_{0+}^{\beta} v(1)\right], I_{0+}^{\beta} v(t)\right) .
\end{aligned}
$$

Since

$$
\begin{aligned}
\left|b I_{0+}^{\alpha} u(\eta)-I_{0+}^{\beta} v(1)\right| \leq & \left|\frac{b}{\Gamma(\alpha)} \int_{0}^{\eta}(\eta-s)^{\alpha-1} u(s) d s\right| \\
& +\left|\frac{1}{\Gamma(\beta)} \int_{0}^{1}(1-s)^{\alpha-1} v(s) d s\right| \\
\leq & \frac{b}{\Gamma(\alpha)}\|u\|_{1}+\frac{1}{\Gamma(\beta)}\|v\|_{1}, \\
D_{0+}^{\alpha-1} I_{0+}^{\alpha} u(t)= & \int_{0}^{t} u(s) d s, \\
I_{0+}^{\alpha} u(t)= & \frac{1}{\Gamma(\alpha)} \int_{0}^{t}(t-s)^{\alpha-1} u(s) d s, \\
D_{0+}^{\beta-1} I_{0+}^{\beta} v(t)= & \int_{0}^{t} v(s) d s, \\
I_{0+}^{\beta} v(t)= & \frac{1}{\Gamma(\beta)} \int_{0}^{t}(t-s)^{\beta-1} v(s) d s,
\end{aligned}
$$

then

$$
\left\|K_{P}(u, v)\right\|_{Y} \leq \Delta_{1}\|(u, v)\|_{Z} .
$$

In fact, if $(u, v) \in \operatorname{Im} L$, then

$$
\begin{aligned}
& L K_{P}(u, v)(t) \\
& =\left\{D_{0+}^{\alpha}\left[I_{0+}^{\alpha} u(t)-a \xi^{\beta-1} t^{\alpha-1}\left[b I_{0+}^{\alpha} u(\eta)-I_{0+}^{\beta} v(1)\right]\right],\right. \\
& \left.\quad D_{0+}^{\beta} I_{0+}^{\beta} v(t)\right\}=(u, v) .
\end{aligned}
$$

By Lemma 4 , for $(u, v) \in \operatorname{dom} L \cap \operatorname{Ker} P$,

$$
\begin{aligned}
& K_{P} L(u, v)(t) \\
& =\left(I_{0+}^{\alpha} D_{0+}^{\alpha} u(t)-a \xi^{\alpha-1} t^{\alpha-1}\left[b I_{0+}^{\alpha} D_{0+}^{\alpha} u(\eta)-I_{0+}^{\beta} D_{0+}^{\beta} v(1)\right],\right. \\
& \left.\quad I_{0+}^{\beta} D_{0+}^{\beta} v(t)\right)
\end{aligned}
$$

$$
\begin{gathered}
=\left(u(t)-\frac{D_{0+}^{\alpha-1} u(0)}{\Gamma(\alpha)} t^{\alpha-1}-\frac{I_{0+}^{2-\alpha} u(0)}{\Gamma(\alpha-1)} t^{\alpha-2}-a \xi^{\beta-1} t^{\alpha-1}\right. \\
\times\left[b u(\eta)-b \frac{D_{0+}^{\alpha-1} u(0)}{\Gamma(\alpha)} \eta^{\alpha-1}-\frac{I_{0+}^{2-\alpha} u(0)}{\Gamma(\alpha-1)} \eta^{\alpha-2}\right. \\
\left.\left.\quad-v(1)+\frac{D_{0+}^{\beta-1} v(0)}{\Gamma(\beta)}\right], v(t)-\frac{D_{0+}^{\beta-1} v(0)}{\Gamma(\beta)} t^{\beta-1}\right) \\
=\left(u(t)-\frac{D_{0+}^{\alpha-1} u(0)}{\Gamma(\alpha)} t^{\alpha-1}\right. \\
\left.\quad+a \xi^{\beta-1} t^{\alpha-1} b \frac{D_{0+}^{\alpha-1} u(0)}{\Gamma(\alpha)} \eta^{\alpha-1}, v(t)\right) \\
=(u(t), v(t))
\end{gathered}
$$

$\left(D_{0+}^{\beta-1} v(0)=0\right.$ since $(u, v) \in \operatorname{Ker} P$ and $I_{0+}^{2-\alpha} u(0)=0$ since $(u, v) \in \operatorname{dom} L)$. Hence,

$$
K_{p}=\left(\left.L\right|_{\operatorname{dom} L \cap \operatorname{Ker} P}\right)^{-1} .
$$

The proof is complete.

\section{Main Results}

In this section, we will use Theorem 1 to prove the existence of solutions to BVP (1). To obtain our main theorem, we use the following assumptions.

(H1) There exist functions $a_{i}, b_{i}, c_{i}, d_{i}, e_{i} \in L^{1}[0,1](i=$ $1,2)$ such that for all $(x, y, z, w) \in \mathbb{R}^{4}, t \in[0,1]$,

$$
\begin{aligned}
|f(t, x, y, z, w)| \leq & e_{1}(t)+a_{1}(t)|x|+b_{1}(t)|y| \\
& +c_{1}(t)|z|+d_{1}(t)|w|, \\
|g(t, x, y, z, w)| \leq & e_{2}(t)+a_{2}(t)|x|+b_{2}(t)|y| \\
& +c_{2}(t)|z|+d_{2}(t)|w| .
\end{aligned}
$$

(H2) There exists a constant $A>0$ such that, for $(u, v) \in$ $\operatorname{dom} L$, if $\left|D_{0+}^{\beta-1} v(t)\right|>A$ for all $t \in[0,1]$, then $Q_{1}\left(N_{1}(u, v)\right) \neq 0$ or $Q_{1}\left(N_{2}(u, v)\right) \neq 0$.

(H3) There exists a constant $B>0$ such that either, for each $c \in \mathbb{R}:|c|>B$,

$c N_{1}\left(c a \xi^{\beta-1} t^{\alpha-1}, c t^{\beta-1}\right)>0, \quad c N_{2}\left(c a \xi^{\beta-1} t^{\alpha-1}, c t^{\beta-1}\right)>0$

or, for each $a \in \mathbb{R}:|a|>B$,

$$
\begin{aligned}
& c N_{1}\left(c a \xi^{\beta-1} t^{\alpha-1}, c t^{\beta-1}\right)<0, \\
& c N_{2}\left(c a \xi^{\beta-1} t^{\alpha-1}, c t^{\beta-1}\right)<0 .
\end{aligned}
$$


Theorem 10. Suppose (2) and (H1)-(H3) hold. Then (1) has at least one solution in $Y$, provided that

$\max \left\{\left(\Delta_{1}+\Delta_{3}\right) \max \left\{\left\|a_{2}\right\|_{1},\left\|b_{2}\right\|_{1},\left\|c_{2}\right\|_{1},\left\|d_{2}\right\|_{1}\right\}, \Delta_{4}, \Delta_{5}\right\}$ $<1$.

Proof. Set

$$
\begin{gathered}
\Omega_{1}=\{(u, v) \in \operatorname{dom} L \backslash \operatorname{Ker} L: L(u, v)=\lambda N(u, v) \\
\text { for some } \lambda \in[0,1]\} .
\end{gathered}
$$

Take $(u, v) \in \Omega_{1}$. Since $L u=\lambda N u$, so $\lambda \neq 0$ and $N(u, v) \in$ $\operatorname{Im} L=\operatorname{Ker} Q$; hence,

$$
Q N(u, v)=Q_{1}\left(N_{1}(u, v), N_{2}(u, v)\right)(1,1)=0 .
$$

Thus, from $(\mathrm{H} 2)$, there exist $t_{0} \in[0,1]$ such that

$$
\left|D_{0+}^{\beta-1} v\left(t_{0}\right)\right| \leq A \text {. }
$$

Noticing that

$$
D_{0+}^{\beta-1} v(t)=D_{0+}^{\beta-1} v\left(t_{0}\right)+\int_{t_{0}}^{t} D_{0+}^{\beta} v(s) d s
$$

so

$$
\begin{aligned}
\left|D_{0+}^{\beta-1} v(0)\right| & \leq\left\|D_{0+}^{\beta-1} v(t)\right\|_{\infty} \leq\left|D_{0+}^{\beta-2} v\left(t_{0}\right)\right|+\left\|D_{0+}^{\beta} v(t)\right\|_{1} \\
& \leq A+\left\|L_{2} v\right\|_{1} \leq A+\left\|N_{2}(u, v)\right\|_{1} .
\end{aligned}
$$

Thus

$$
\begin{aligned}
\|P(u, v)\|_{Y} & =\left\|\frac{D_{0+}^{\beta-1} v(0)}{\Gamma(\beta)}\left(a \xi^{\beta-1} t^{\alpha-1}, t^{\beta-1}\right)\right\|_{Y} \\
& =\Delta_{3}\left|D_{0+}^{\beta-1} v(0)\right| \leq \Delta_{3}\left(A+\left\|N_{2}(u, v)\right\|_{1}\right) .
\end{aligned}
$$

For all $(u, v) \in \Omega_{1},(I-P)(u, v) \in \operatorname{dom} L \cap$ Ker $P$. Considering Lemma 9, we get $L P(u, v)=(0,0)$. Together with (39), we have

$$
\begin{aligned}
\|(I-P)(u, v)\|_{Y} & =\left\|K_{P} L(I-P)(u, v)\right\|_{Y} \\
& \leq \Delta_{1}\|L(I-P)(u, v)\|_{Z} \\
& \leq \Delta_{1}\|N(u, v)\|_{Z} .
\end{aligned}
$$

From (60) and (61), we have

$\|(u, v)\|_{Y}$

$$
\begin{gathered}
\leq\|P(u, v)\|_{Y}+\|(I-P)(u, v)\|_{Y} \\
\leq \Delta_{3}\left(A+\left\|N_{2}(u, v)\right\|_{1}\right)+\Delta_{1}\|N(u, v)\|_{Z} \\
=A \Delta_{3}+\max \left\{\left(\Delta_{1}+\Delta_{3}\right)\left\|N_{2}(u, v)\right\|_{1}, \Delta_{1}\left\|N_{1}(u, v)\right\|_{1}\right. \\
\left.+\Delta_{3}\left\|N_{2}(u, v)\right\|_{1}\right\} .
\end{gathered}
$$

From (62), we discuss various cases.

Case $1\left(\|(u, v)\|_{Y} \leq A \Delta_{3}+\left(\Delta_{1}+\Delta_{3}\right)\left\|N_{2}(u, v)\right\|_{1}\right)$. From (H1), we have

$$
\begin{aligned}
\|(u, v)\|_{Y} & \\
\leq & A \Delta_{3}+\left(\Delta_{1}+\Delta_{3}\right) \\
& \times\left(\left\|a_{2}\right\|_{1}\|u\|_{\infty}+\left\|b_{2}\right\|_{1}\left\|D_{0+}^{\alpha-1} u\right\|_{\infty}+\left\|c_{2}\right\|_{1}\|v\|_{\infty}\right. \\
& \left.+\left\|d_{2}\right\|_{1}\left\|D_{0+}^{\beta-1} v\right\|_{\infty}+\left\|e_{2}\right\|_{1}\right) \\
\leq & A \Delta_{3}+\left(\Delta_{1}+\Delta_{3}\right)\left\|e_{2}\right\|_{1} \\
& +\left(\Delta_{1}+\Delta_{3}\right) \max \left\{\left\|a_{2}\right\|_{1},\left\|b_{2}\right\|_{1}\right\}\|u\|_{C^{\alpha-1}} \\
+ & \left(\Delta_{1}+\Delta_{3}\right) \max \left\{\left\|c_{2}\right\|_{1},\left\|d_{2}\right\|_{1}\right\}\|v\|_{C^{\beta-1}} \\
\leq & A \Delta_{3}+\left(\Delta_{1}+\Delta_{3}\right)\left\|e_{2}\right\|_{1} \\
+ & \left(\Delta_{1}+\Delta_{3}\right) \max \left\{\left\|a_{2}\right\|_{1},\left\|b_{2}\right\|_{1},\left\|c_{2}\right\|_{1},\left\|d_{2}\right\|_{1}\right\}\|(u, v)\|_{Y},
\end{aligned}
$$

which yield

$\|(u, v)\|_{Y}$

$$
\leq \frac{A \Delta_{2}+\left(\Delta_{1}+\Delta_{3}\right)\left\|e_{2}\right\|_{1}}{1-\left(\Delta_{1}+\Delta_{3}\right) \max \left\{\left\|a_{2}\right\|_{1},\left\|b_{2}\right\|_{1},\left\|c_{2}\right\|_{1},\left\|d_{2}\right\|_{1}\right\}} .
$$

Thus, $\Omega_{1}$ is bounded.

Case $2\left(\|(u, v)\|_{Y} \leq A \Delta_{3}+\Delta_{1}\left\|N_{1}(u, v)\right\|_{1}+\Delta_{3}\left\|N_{2}(u, v)\right\|_{1}\right)$. From (H1), we have

$\|(u, v)\|_{Y}$

$$
\begin{gathered}
\leq A \Delta_{3}+\Delta_{1}\left(\left\|a_{1}\right\|_{1}\|u\|_{\infty}+\left\|b_{1}\right\|_{1}\left\|D_{0+}^{\alpha-1} u\right\|_{\infty}+\left\|c_{1}\right\|_{1}\|v\|_{\infty}\right. \\
\left.+\left\|d_{1}\right\|_{1}\left\|D_{0+}^{\beta-1} v\right\|_{\infty}+\left\|e_{1}\right\|_{1}\right) \\
+\Delta_{3}\left(\left\|a_{2}\right\|_{1}\|u\|_{\infty}+\left\|b_{2}\right\|_{1}\left\|D_{0+}^{\alpha-1} u\right\|_{\infty}+\left\|c_{2}\right\|_{1}\|v\|_{\infty}\right. \\
\left.+\left\|d_{2}\right\|_{1}\left\|D_{0+}^{\beta-1} v\right\|_{\infty}+\left\|e_{2}\right\|_{1}\right) \\
\leq A \Delta_{3}+\Delta_{1}\left\|e_{1}\right\|_{1}+\Delta_{3}\left\|e_{2}\right\|_{1}+\Delta_{4}\|u\|_{C^{\alpha-1}}+\Delta_{5}\|v\|_{C^{\beta-1}} \\
\leq A \Delta_{3}+\Delta_{1}\left\|e_{1}\right\|_{1}+\Delta_{3}\left\|e_{2}\right\|_{1}+\max \left\{\Delta_{4}, \Delta_{5}\right\}\|(u, v)\|_{Y}
\end{gathered}
$$

which yield

$$
\|(u, v)\|_{Y} \leq \frac{A \Delta_{3}+\Delta_{1}\left\|e_{1}\right\|_{1}+\Delta_{3}\left\|e_{2}\right\|_{1}}{1-\max \left\{\Delta_{4}, \Delta_{5}\right\}} .
$$

Thus, $\Omega_{1}$ is bounded. Let

$$
\Omega_{2}=\{(u, v) \in \operatorname{Ker} L: N(u, v) \in \operatorname{Im} L\} .
$$

For $(u, v) \in \Omega_{2}$ and $(u, v) \in \operatorname{Ker} L$, so $(u, v)=c\left(a \xi^{\beta-1} t^{\alpha-1}\right.$, $\left.t^{\beta-1}\right), t \in[0,1], c \in \mathbb{R}$. Noticing that $\operatorname{Im} L=\operatorname{Ker} Q$, then we 
get $Q N(u, v)=0$, and thus $Q_{1} N_{1}\left(c a \xi^{\beta-1} t^{\alpha-1}, c t^{\beta-1}\right)=0$ and $Q_{1} N_{2}\left(c a \xi^{\beta-1} t^{\alpha-1}, c t^{\beta-1}\right)=0$. From $(\mathrm{H} 2)$, we get $|c| \leq A / \Gamma(\beta)$, and thus $\Omega_{2}$ is bounded.

We define the isomorphism $J: \operatorname{Ker} L \rightarrow \operatorname{Im} Q$ by

$$
J\left(c a \xi^{\beta-1} t^{\alpha-1}, c t^{\beta-1}\right)=(c, c) \text {. }
$$

If the first part of (H3) is satisfied, then let

$$
\begin{aligned}
& \Omega_{3}=\{(u, v) \in \operatorname{ker} L: \lambda J(u, v) \\
&+(1-\lambda) Q N(u, v)=0, \lambda \in[0,1]\} .
\end{aligned}
$$

For $(u, v)=\left(c a \xi^{\beta-1} t^{\alpha-1}, c t^{\beta-1}\right) \in \Omega_{3}$,

$$
\lambda(c, c)=-(1-\lambda) \Delta_{2} Q_{1} N\left(c a \xi^{\beta-1} t^{\alpha-1}, c t^{\beta-1}\right)(1,1) .
$$

If $\lambda=1$, then $c=0$. Otherwise, if $|c|>B$, in view of (H3) and $\Delta_{2}<0$, one has

$$
(1-\lambda) \Delta_{3} Q_{1} N\left(c a \xi^{\beta-1} t^{\alpha-1}, c t^{\beta-1}\right)<0
$$

which contradict $\lambda c^{2} \geq 0$. Thus $\Omega_{3} \subset\{(u, v) \in \operatorname{Ker} L \mid(u, v)=$ $\left.\left(c a \xi^{\beta-1} t^{\alpha-1}, c t^{\beta-1}\right),|c| \leq B\right\}$ is bounded.

If the second part of (H3) holds, then define the set

$$
\begin{aligned}
& \Omega_{3}=\{(u, v) \in \operatorname{Ker} L:-\lambda J(u, v) \\
&+(1-\lambda) Q N(u, v)=0, \lambda \in[0,1]\},
\end{aligned}
$$

and here $J$ is as above. Similar to the above argument, we can show that $\Omega_{3}$ is bounded too.

In the following, we will prove that all conditions of Theorem 1 are satisfied. Let $\Omega$ be a bounded open subset of $Y$ such that $\mathrm{U}_{i=1}^{3} \overline{\Omega_{i}} \subset \Omega$. By standard arguments, we can prove that $K_{P}(I-Q) N: \Omega \rightarrow Y$ is compact, and thus $N$ is $L$ compact on $\bar{\Omega}$. Then by the above argument we have

(i) $L u \neq \lambda N u$, for every $(u, \lambda) \in[(\operatorname{dom} L \backslash \operatorname{Ker} L) \cap \partial \Omega] \times$ $(0,1)$

(ii) $N u \notin \operatorname{Im} L$ for $u \in \operatorname{Ker} L \cap \partial \Omega$.

Finally, we will prove that (iii) of Theorem 1 is satisfied. Let $H(u, \lambda)= \pm \lambda J u+(1-\lambda) Q N u$. According to the above argument, we know that

$$
H(u, \lambda) \neq 0 \text { for } u \in \operatorname{Ker} L \cap \partial \Omega \text {. }
$$

Thus, by the homotopy property of degree,

$$
\begin{aligned}
\operatorname{deg} & \left(\left.Q N\right|_{\operatorname{Ker} L}, \operatorname{Ker} L \cap \Omega, 0\right) \\
= & \operatorname{deg}(H(\cdot, 0), \operatorname{Ker} L \cap \Omega, 0) \\
= & \operatorname{deg}(H(\cdot, 1), \operatorname{Ker} L \cap \Omega, 0) \\
= & \operatorname{deg}( \pm J, \operatorname{Ker} L \cap \Omega, 0) \neq 0 .
\end{aligned}
$$

Then by Theorem $1, L(u, v)=N(u, v)$ has at least one solution in $\operatorname{dom} L \cap \bar{\Omega}$ so that BVP (1) has a solution in $C^{\alpha-1}[0,1] \times$ $C^{\beta-1}[0,1]$. The proof is complete.

\section{Conflict of Interests}

The authors declare that there is no conflict of interests regarding the publication of this paper.

\section{Acknowledgments}

The authors are very grateful to the anonymous referees for many valuable comments and suggestions which helped to improve the presentation of the paper. The project was supported by the National Natural Science Foundation of China (11371221, 61304074), the Specialized Research Foundation for the Doctoral Program of Higher Education of China (20123705110001), the Program for Scientific Research Innovation Team in Colleges and Universities of Shandong Province, the Postdoctoral Science Foundation of Shandong Province (201303074), and Foundation of SDUST.

\section{References}

[1] A. A. Kilbas, H. M. Srivastava, and J. J. Trujillo, Theory and Applications of Fractional Differential Equations, vol. 204 of North-Holland Mathematics Studies, Elsevier Science B.V., Amsterdam, The Netherlands, 2006.

[2] V. Lakshmikantham, S. Leela, and J. Vasundhara Devi, Theory of Fractional Dynamic Systems, Cambridge Academic Publishers, Cambridge, UK, 2009.

[3] J. J. Nieto and J. Pimentel, "Positive solutions of a fractional thermostat model," Boundary Value Problems, vol. 2013, article 5, 2013.

[4] I. Podlubny, Fractional Differential Equations, vol. 198, Academic Press, San Diego, Calif, USA, 1999.

[5] W. Wu and X. Zhou, "Eigenvalue of fractional differential equations with P-Laplacian operator," Discrete Dynamics in Nature and Society, vol. 2013, Article ID 137890, 8 pages, 2013.

[6] X. Zhang, L. Liu, Y. Wu, and Y. Lu, "The iterative solutions of nonlinear fractional differential equations," Applied Mathematics and Computation, vol. 219, no. 9, pp. 4680-4691, 2013.

[7] X. Zhang, L. Liu, and Y. Wu, "The uniqueness of positive solution for a singular fractional differential system involving derivatives," Communications in Nonlinear Science and Numerical Simulation, vol. 18, no. 6, pp. 1400-1409, 2013.

[8] X. Zhang, L. Liu, and Y. Wu, "The eigenvalue problem for a singular higher order fractional differential equation involving fractional derivatives," Applied Mathematics and Computation, vol. 218, no. 17, pp. 8526-8536, 2012.

[9] X. Zhang, L. Liu, and Y. Wu, "Existence results for multiple positive solutions of nonlinear higher order perturbed fractional differential equations with derivatives," Applied Mathematics and Computation, vol. 219, no. 4, pp. 1420-1433, 2012.

[10] X. Zhang, L. Liu, B. Wiwatanapataphee, and Y. Wu, "Positive solutions of eigenvalue problems for a class of fractional differential equations with derivatives," Abstract and Applied Analysis, vol. 2012, Article ID 512127, 16 pages, 2012.

[11] X. Zhang, L. Liu, and Y. Wu, "Multiple positive solutions of a singular fractional differential equation with negatively perturbed term," Mathematical and Computer Modelling, vol. 55, no. 3-4, pp. 1263-1274, 2012.

[12] K. Deng, "Blow-up rates for parabolic systems," Zeitschrift für Angewandte Mathematik und Physik, vol. 47, no. 1, pp. 132-143, 1996. 
[13] K. Deng, "Global existence and blow-up for a system of heat equations with non-linear boundary conditions," Mathematical Methods in the Applied Sciences, vol. 18, no. 4, pp. 307-315, 1995.

[14] L. Zhigui and X. Chunhong, "The blow-up rate for a system of heat equations with nonlinear boundary conditions," Nonlinear Analysis. Theory, Methods \& Applications, vol. 34, no. 5, pp. 767778, 1998.

[15] D. G. Aronson, "A comparison method for stability analysis of nonlinear parabolic problems," SIAM Review, vol. 20, no. 2, pp. 245-264, 1978.

[16] M. Pedersen and Z. Lin, "Blow-up analysis for a system of heat equations coupled through a nonlinear boundary condition," Applied Mathematics Letters, vol. 14, no. 2, pp. 171-176, 2001.

[17] N. A. Asif and R. A. Khan, "Positive solutions to singular system with four-point coupled boundary conditions," Journal of Mathematical Analysis and Applications, vol. 386, no. 2, pp. 848-861, 2012.

[18] C. Yuan, D. Jiang, D. O’Regan, and R. P. Agarwal, "Multiple positive solutions to systems of nonlinear semipositone fractional differential equations with coupled boundary conditions," Electronic Journal of Qualitative Theory of Differential Equations, no. 13, p. 17, 2012.

[19] Y. Cui and J. Sun, "On existence of positive solutions of coupled integral boundary value problems for a nonlinear singular superlinear differential system," Electronic Journal of Qualitative Theory of Differential Equations, no. 41, pp. 1-13, 2012.

[20] Z. Bai and Y. Zhang, "Solvability of fractional three-point boundary value problems with nonlinear growth," Applied Mathematics and Computation, vol. 218, no. 5, pp. 1719-1725, 2011.

[21] Y. Cui, "Solvability of second-order boundary-value problems at resonance involving integral conditions," Electronic Journal of Differential Equations, no. 45, pp. 1-9, 2012.

[22] Z. Hu and W. Liu, "Solvability for fractional order boundary value problems at resonance," Boundary Value Problems, vol. 2011, article 20, 2011.

[23] W. Jiang, "The existence of solutions to boundary value problems of fractional differential equations at resonance," Nonlinear Analysis. Theory, Methods \& Applications, vol. 74, no. 5, pp. 1987-1994, 2011.

[24] N. Kosmatov, "Multi-point boundary value problems on time scales at resonance," Journal of Mathematical Analysis and Applications, vol. 323, no. 1, pp. 253-266, 2006.

[25] G. Wang, W. Liu, S. Zhu, and T. Zheng, "Existence results for a coupled system of nonlinear fractional $2 \mathrm{~m}$-point boundary value problems at resonance," Advances in Difference Equations, vol. 2011, article 80, 2011.

[26] J. R. L. Webb, "Remarks on nonlocal boundary value problems at resonance," Applied Mathematics and Computation, vol. 216, no. 2, pp. 497-500, 2010.

[27] J. R. L. Webb and M. Zima, "Multiple positive solutions of resonant and non-resonant non-local fourth-order boundary value problems," Glasgow Mathematical Journal, vol. 54, no. 1, pp. 225-240, 2012.

[28] X. Zhang, M. Feng, and W. Ge, "Existence result of second-order differential equations with integral boundary conditions at resonance," Journal of Mathematical Analysis and Applications, vol. 353, no. 1, pp. 311-319, 2009.

[29] X. Zhang, C. Zhu, and Z. Wu, "Solvability for a coupled system of fractional differential equations with impulses at resonance," Boundary Value Problems, vol. 2013, artice 80, 2013.
[30] Z. Zhao and J. Liang, "Existence of solutions to functional boundary value problem of second-order nonlinear differential equation," Journal of Mathematical Analysis and Applications, vol. 373, no. 2, pp. 614-634, 2011.

[31] Y. Zou and Y. Cui, "Existence results for a functional boundary value problem of fractional differential equations," Advances in Difference Equations, vol. 2013, article 25, 2013.

[32] J. Mawhin, “Topological degree and boundary value problems for nonlinear differential equations," in Topological Methods for Ordinary Differential Equations, P. M. Fitzpertrick, M. Martelli, J. Mawhin, and R. Nussbaum, Eds., vol. 1537 of Lecture Notes in Mathematics, pp. 74-142, Springer, Berlin, Germany, 1993.

[33] J. Mawhin, Topological Degree Methods in Nonlinear Boundary Value Problems, vol. 40 of CBMS Regional Conference Series in Mathematics, American Mathematical Society, Providence, RI, USA, 1979.

[34] Y. Zhang and Z. Bai, "Existence of solutions for nonlinear fractional three-point boundary value problems at resonance," Journal of Applied Mathematics and Computing, vol. 36, no. 1-2, pp. 417-440, 2011. 


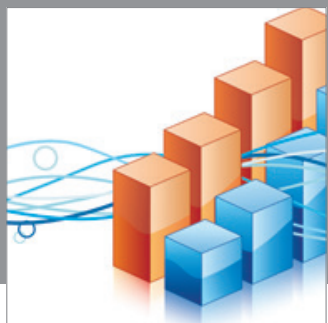

Advances in

Operations Research

mansans

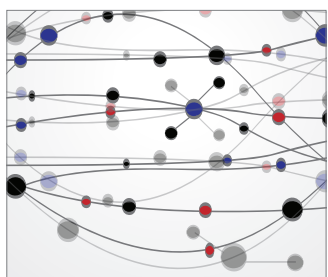

The Scientific World Journal
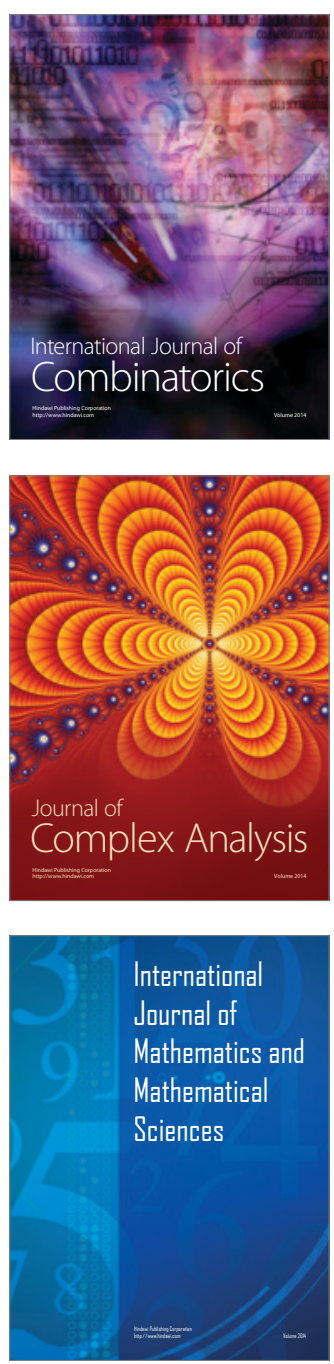
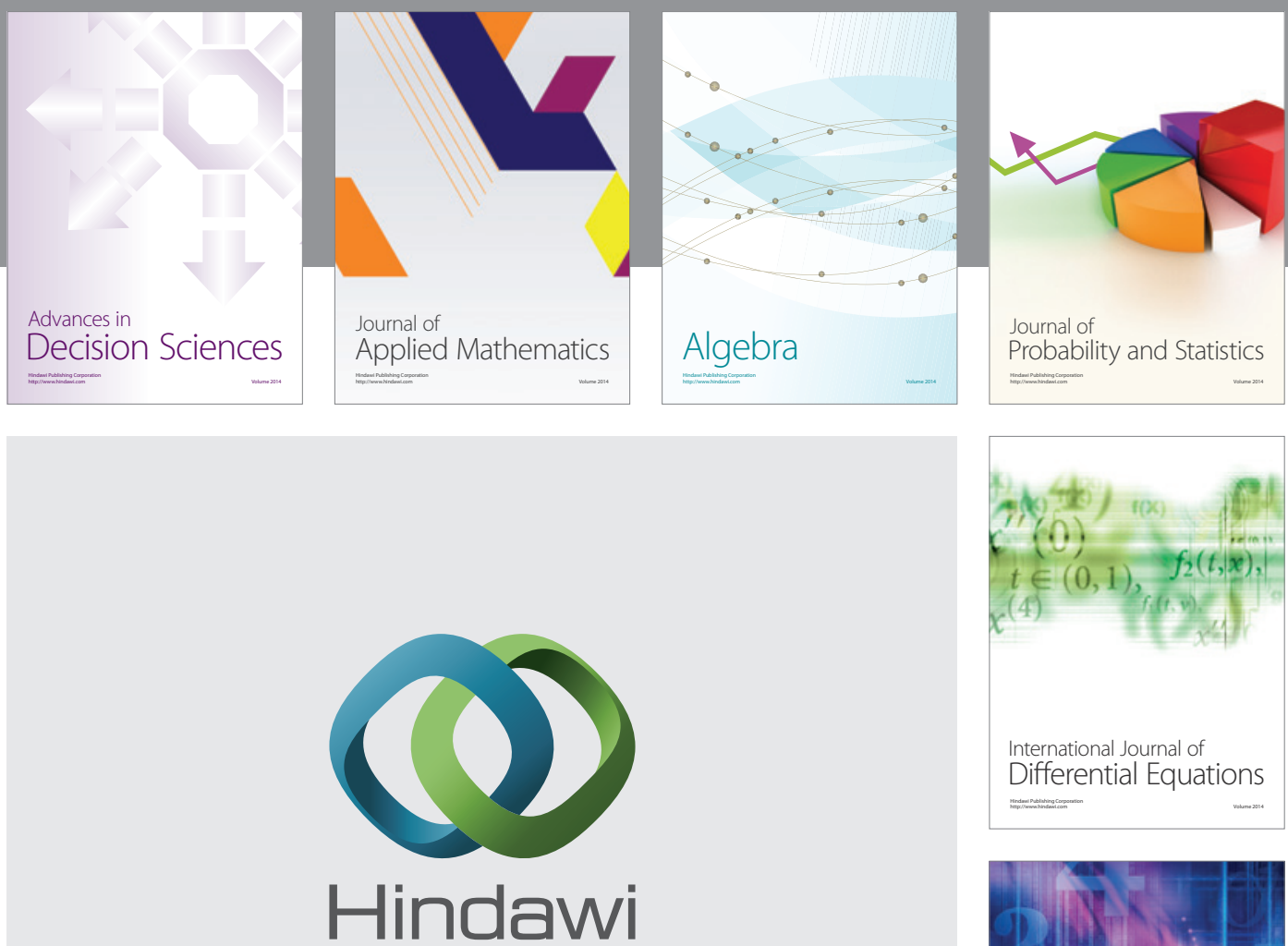

Submit your manuscripts at http://www.hindawi.com
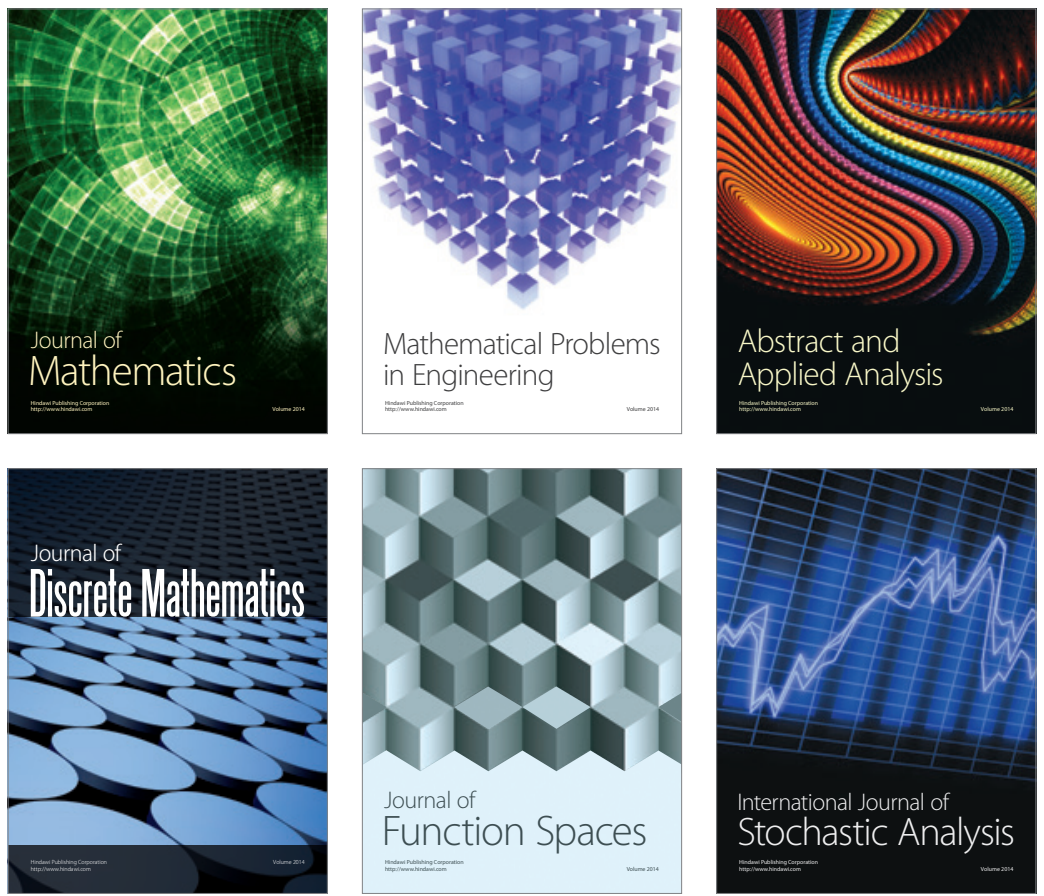

Journal of

Function Spaces

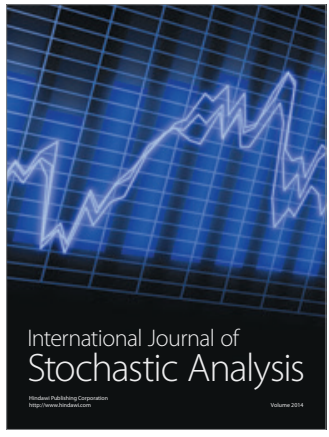

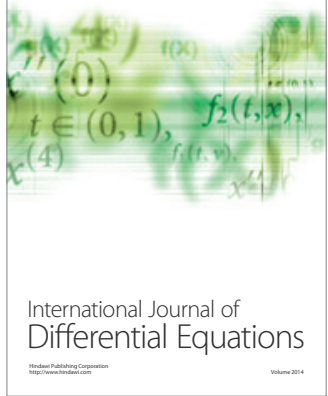
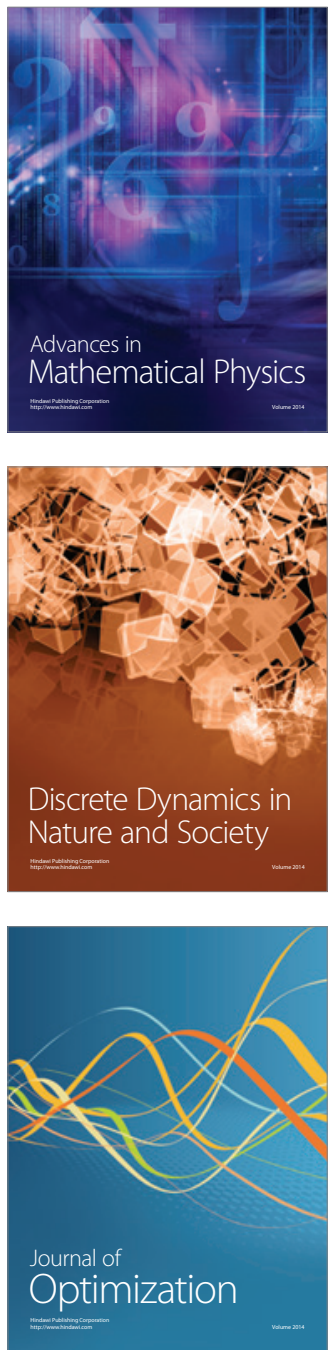